\title{
Perfil clínico-epidemiológico dos casos de tuberculose com coinfecção HIV no município de Belém
}

\author{
Clinical-epidemiological profile of tuberculosis cases with HIV coinfection in the city of \\ Belém
}

\section{Perfil clínico-epidemiológico de los casos de tuberculosis con coinfección por VIH en el municipio de Belém}

Wellen Sampaio Ferreira ${ }^{1 *}$, Gabriel Gonçalves Avelar², Felipe Xavier de $\mathrm{Melo}^{2}$, Mario Carlos Soares Moraes Neto ${ }^{2}$, Tales Sarmento Krischer ${ }^{1}$, Arilson Lima da Silva ${ }^{1}$, Jéssika Sayuri Campelo Kato ${ }^{1}$, Santino Carvalho Franco ${ }^{1}$.

\section{RESUMO}

Objetivo: Traçar o perfil clínico-epidemiológico da coinfecção por tuberculose e HIV no município de Belém. Métodos: Estudo epidemiológico descritivo, realizado por meio dos dados provenientes do Sistema de Informação de Agravos de Notificação (SINAN), no município de Belém, no período de 2009 a 2019. As variáveis analisadas foram: faixa etária, gênero, raça, escolaridade, tipo de entrada, forma clínica, realização do Tratamento Diretamente Observado (TDO), realização da Terapia Antirretroviral (TARV), situação de encerramento e teste sorológico para detecção do HIV. Resultados: Foram notificados 20054 casos de tuberculose no período, dos quais, 2596 (12,94\%) eram de coinfectados por tuberculose e HIV. O perfil dos indivíduos coinfectados segue o seguinte padrão: caso novo (79,08\%), gênero masculino $(69,79 \%)$, ensino fundamental incompleto $(32,81 \%)$, idade entre 20 a 39 anos $(59,89 \%)$, raça parda (77,65\%), forma pulmonar (63,25\%) e TDO não realizado (51,54\%). Ademais, $71,49 \%$ das notificações não contavam com informações acerca da realização da TARV. A cura foi o principal desfecho clínico $(46,64 \%)$. Ao longo dos anos, o percentual de pacientes com tuberculose não testados para infecção pelo HIV diminuiu. Conclusão: Ao caracterizar o perfil da população afetada, este estudo contribui com a implantação de estratégias para a prevenção e controle de ambas as doenças no município.

Palavras-chave: Tuberculose, HIV, Epidemiologia.

\begin{abstract}
Objective: To trace the clinical-epidemiological profile of coinfection with tuberculosis and HIV in the city of Belém. Methods: Descriptive epidemiological study, carried out using data from the Notifiable Diseases Information System (SINAN), in the city of Belém, during the period from 2009 to 2019. The variables analyzed were: age group, gender, race, education, type of entry, clinical form, performance of Directly Observed Treatment (DOT), performance of Antiretroviral Therapy (ART), outcome of the cases and request for serological test for HIV detection. Results: 20054 tuberculosis cases were reported in the period, of which $2596(12,94 \%)$ were coinfected with tuberculosis and HIV. The profile of coinfected individuals follows the following pattern: new case $(79,08 \%)$, male gender $(69,79 \%)$, incomplete primary education $(32,81 \%)$, age between 20 to 39 years $(58,89 \%)$, brown race $(77,65 \%)$, pulmonary form $(63,25 \%)$ and unrealized DOT $(51,54 \%)$. In addition, $71,49 \%$ of the notifications did not have information about performing ART. The cure was the main clinical outcome $(46,64 \%)$. Over the years, the percentage of tuberculosis patients not tested for HIV infection has decreased. Conclusion: By characterizing the profile of the affected population, this study contributes to the implementation of strategies for the prevention and control of both diseases in the city.
\end{abstract}

Keywords: Tuberculosis, HIV, Epidemiology.

1 Universidade do Estado do Pará (UEPA), Belém - PA. E-mail*: sampaiowellen21@gmail.com

2 Universidade da Amazônia (UNAMA), Belém - PA. 


\section{RESUMEN}

Objetivo: Rastrear el perfil clínico-epidemiológico de coinfección con tuberculosis y VIH en el municipio de Belém. Métodos: Estudio epidemiológico descriptivo, utilizando datos del Sistema de Información de Enfermedades de Notificación (SINAN), en el municipio de Belém, durante el período de 2009 a 2019. Las variables analizadas fueron: edad, género, raza, escolaridad, tipo de ingreso, forma clínica, desempeño del tratamiento directamente observado (TDO), desempeño del tratamiento antirretroviral (TARV), el cierre de los casos y prueba serológica para la detección del VIH. Resultados: Se notificaron 20054 casos de tuberculosis en el período, de los cuales 2596 (12,94\%) estaban coinfectados con tuberculosis y VIH. El perfil de las personas coinfectadas sigue el siguiente patrón: nuevo caso $(79,08 \%)$, género masculino $(69,79 \%)$, educación primaria incompleta (32,81\%), edad entre 20 y 39 años $(59,89 \%)$, raza parda $(77,65 \%)$, forma pulmonar $(63,25 \%)$ y TDO no realizado (51,54\%). Además, $71,49 \%$ de las notificaciones no tenían información sobre el desempeño del TARV. La cura fue el principal resultado (46,64\%). Con los años, el porcentaje de pacientes con tuberculosis que no hicieron la prueba de infección por VIH ha disminuido. Conclusión: Al caracterizar el perfil de la población afectada, este estudio contribuye a la implementación de estrategias de prevención y control de ambas enfermedades en el municipio.

Palabras clave: Tuberculosis, VIH, Epidemiología.

\section{INTRODUÇÃO}

A Tuberculose (TB) ainda representa um sério e desafiador problema de saúde pública global. Em âmbito mundial, no ano de 2018, cerca de dez milhões de pessoas adoeceram por tuberculose e 1,5 milhão de pessoas morreram em decorrência dela, sendo a TB a principal causa de morte por um único agente infeccioso (WHO, 2019). O Brasil, com coeficiente de incidência anual girando em torno de 35 casos por 100 mil habitantes, responde por aproximadamente um terço dos casos notificados nas Américas, integrando a lista dos 22 países com maior carga da doença no mundo (BRASIL, 2019; PAHO, 2014).

Além de continuar endêmica nos países em desenvolvimento, a tuberculose ganhou força com 0 surgimento da AIDS, sendo a terceira doença oportunista mais prevalente em portadores do HIV (BENITO $\mathrm{N}$, et al., 2011). O HIV não só tem contribuído para um crescente número de casos de TB, como também tem sido um dos principais responsáveis pelo aumento da mortalidade, o que pode ser evidenciado pelo registro de 400 mil mortes, no ano de 2015, entre os indivíduos coinfectados (BARBOSA IR e COSTA ICC, 2014; WHO, 2017).

A interação entre o HIV e o Mycobacterium tuberculosis é complexa. A tuberculose pode causar elevação da carga viral e redução da contagem de CD4+ em pessoas que vivem com HIV. Por outro lado, a soropositividade ao HIV também pode modificar a patogênese da TB, levando a baciloscopias negativas, achados de imagem atípicos e manifestações extrapulmonares, tornando difícil o diagnóstico desta doença; (MONTALES MT, et al., 2015).

Em pessoas coinfectadas por tuberculose e HIV, a forma extrapulmonar frequentemente ocorre devido à supressão imunológica associada a níveis de CD4 abaixo de 500 células $/ \mathrm{mm}^{3}$, o que viabiliza a disseminação do Mycobacterium tuberculosis para outros órgãos. Há, desta forma, uma maior probabilidade de evolução para formas graves de TB, a exemplo da TB meníngea, óssea, miliar, entre outras, que contribuem expressivamente para o aumento da morbimortalidade da TB em pacientes infectados pelo HIV. Em contraste, é observada uma maior frequência da forma pulmonar em indivíduos com contagem de CD4 elevada, sem diferenças na apresentação clínica em comparação à população em geral (EFSEN AMW, et al., 2014).

Ademais, os portadores do HIV são aproximadamente 26 vezes mais propensos a desenvolver TB quando comparados aos indivíduos que não estão infectados pelo vírus, de forma que a testagem para o HIV tornou-se recomendação padrão para todos os pacientes com tuberculose (OLIVEIRA LB, et al., 2018; BASTOS SH, et al., 2020). Esta conduta é imprescindível, uma vez que permite o diagnóstico precoce da infecção pelo HIV, possibilitando, desta maneira, o início da terapia antirretroviral, que por conseguinte levará à diminuição de sua morbidade e mortalidade, além de representar uma importante ferramenta para a elaboração e execução de políticas públicas (MIRANDA LO, et al., 2017). 
Ainda, é importante salientar que a dupla carga de doenças dificulta a adesão ao tratamento, no qual o indivíduo possui uma predisposição ao abandono do tratamento da tuberculose, em virtude das reações adversas e interações medicamentosas serem mais intensas em pacientes soropositivos, levando, em muitos casos, ao uso prioritário dos antirretrovirais em detrimento do medicamento para TB. Evidencia-se, portanto, o elevado perigo da coinfecção, visto que as altas taxas de abandono encontradas neste grupo contribuem de sobremaneira para a multirresistência aos medicamentos (WHO, 2017; OLIVEIRA LB, et al., 2018).

Neste cenário, faz-se necessário o manejo de ambas as doenças de forma articulada e integral, respeitando as particularidades dos indivíduos que apresentam a coinfecção, e garantindo que a responsabilidade seja compartilhada entre os programas de controle, a fim de minimizar a ocorrência desta associação. Para tanto, destacam-se algumas estratégias fundamentais para o controle da coinfecção TB/HIV, como o diagnóstico precoce de ambas as doenças e a garantia de um tratamento adequado e realizado de maneira oportuna (BASTOS SH, et al., 2019). Para este fim, foi proposta a implementação do Tratamento Diretamente Observado (TDO), uma estratégia que consiste na observação da tomada dos medicamentos por um profissional de saúde treinado, objetivando reduzir ou até mesmo eliminar os casos de abandono do tratamento e garantir a tomada correta da medicação (OLIVEIRA LB, et al., 2018).

Outro ponto concernente à temática é em relação ao estigma apresentado historicamente pela tuberculose e pelo HIV. Sentimentos de culpa, rejeição e exclusão por conta da condição de saúde particular corroboram com comportamentos de isolamento social e até mesmo com o isolamento de pessoas próximas, tais como familiares e amigos. Por outro lado, nota-se que no atual estágio da epidemia de TB/HIV, avanços importantes foram alcançados na forma como tais enfermidades são vistas pela comunidade, o que é resultado do maior conhecimento adquirido pela população. No entanto, o preconceito ainda se faz presente em uma parcela significativa da sociedade, o que interfere de forma negativa no processo saúde-doença. Além do mais, é importante ressaltar que o preconceito existente não se restringe somente àquele advindo da popução em geral, mas também envolve o preconceito advindo do próprio indivíduo, o qual está embasado em uma concepção de desvalorização moral e social (NEVES LAS, et al., 2012).

Ainda, outro fato relevante é que, entre os anos de 2000 a 2011, as taxas de mortalidade causadas pela coinfecção TB/HIV no Brasil foram mais elevadas em indivíduos do sexo masculino, de cor/raça negra, com idade na faixa economicamente ativa e habitantes da região Sul do país. Em contrapartida, observou-se que a diminuição da mortalidade ocorreu de forma heterogênea entre as regiões brasileiras, o que pode ter relação com o uso da Terapia antirtetroviral (TARV) e atualização dos protocolos utilizados no manejo clínico da coinfecção (BASTOS SH, et al., 2019).

É fundamental pontuar, ainda, que o estado de São Paulo concentra o maior número de casos de TB e Aids do Brasil e apresenta cerca de $9,9 \%$ de casos de TB com coinfecção por HIV (SOUZA AC, et al., 2020). No que concerne a região Norte, esta registrou 1204 casos de coinfecção TB/HIV no ano de 2019. $O$ município de Belém, que notificou 264 novos casos de coinfecção, destacou-se como a segunda capital mais atingida da região (SINAN, 2019). Logo, torna-se imprescindível a realização de trabalhos epidemiológicos voltados a este município, para que, então, torne-se mais compreensível a distribuição da doença nesta localidade. Diante disso, este estudo teve como objetivo traçar o perfil clínico-epidemiológico da coinfecção por tuberculose e HIV no município de Belém.

\section{MÉTODOS}

Trata-se de um estudo epidemiológico descritivo, realizado por meio dos dados extraídos do Sistema de Informações de Agravos de Notificação (SINAN), disponibilizados por intermédio do Departamento de Informática do Sistema Único de Saúde (DATASUS). A pesquisa apresentou como unidade de análise o município de Belém, localizado no Estado do Pará. Este município possui densidade demográfica de 1.315,26 habitantes $/ \mathrm{km}^{2}$, Índice de Desenvolvimento Humano Municipal (IDHM) de 0,746, e apresenta uma população de 1.499.641 habitantes (IBGE, 2010; IBGE 2020). 
A população do estudo foi composta pelos casos de coinfecção por tuberculose e HIV notificados no município de Belém entre os anos de 2009 a 2019. Todos os casos notificados por tuberculose que possuíam o agravo "Aids" confirmado ou sorologia positiva para HIV foram considerados como coinfecção por tuberculose e HIV. Foram excluídos da pesquisa os casos cuja variável "HIV" foi preenchida como negativa, não realizada, em andamento ou deixada em branco.

Para construção do estudo foram investigadas variáveis sociodemográficas, epidemiológicas e clínicas. As variáveis sociodemográficas incluídas, foram: faixa etária, gênero, raça e escolaridade. As variáveis epidemiológicas e clínicas analisadas, foram: tipo de entrada, forma clínica, realização do Tratamento Diretamente Observado (TDO), realização da Terapia Antirretroviral (TARV), situação de encerramento e solicitação de teste sorológico para detecção do HIV.

A variável tipo de entrada foi classificada em caso novo (nunca realizou o tratamento da doença), recidiva (realizou o tratamento, foi curado e, posteriomente, infectado novamente por tuberculose ou casos de persistência de bacilos da tuberculose em pacientes curados), reingresso após abandono (iniciou o tratamento para TB, porém abandonou e logo depois, reiniciou) ou transferência (indivíduo estava seguindo o tratamento em outro serviço de saúde e foi transferido). A variável "situação de encerramento do caso" corresponde ao desfecho do caso após o acompanhamento do tratamento para tuberculose, no qual têmse: cura, abandono, transferência, óbito ou TB Droga Resistente (TB DR).

Por fim, a análise dos dados foi baseada em referenciais teóricos indexados na Biblioteca Virtual de Saúde (BVS) e em bibliografias do Ministério da Saúde. Os descritores em português utilizados, foram: tuberculose, HIV e epidemiologia. Os dados foram tratados por meio de estatística descritiva e apresentados em gráficos e tabelas através dos softwares Microsoft Office Excel 2016 e TabWin. Por se tratar de um banco de domínio público, não foi necessário submeter o projeto ao Comitê de Ética em Pesquisa (CEP).

\section{RESULTADOS}

No período de 2009 a 2019, o município de Belém notificou 20054 casos de tuberculose, dos quais 2596 (12,94\%) estavam coinfectados por TB/HIV. Em 2017, o número de indivíduos diagnosticados foi o maior, com um total de 273 casos, enquanto o menor quantitativo ocorreu em 2009, com 203 casos, demonstrando aumento heterogêneo da prevalência dessa coinfecção (Gráfico 1).

Gráfico 1 - Distribuição do HIV nos casos de Tuberculose em relação ao ano de diagnóstico.

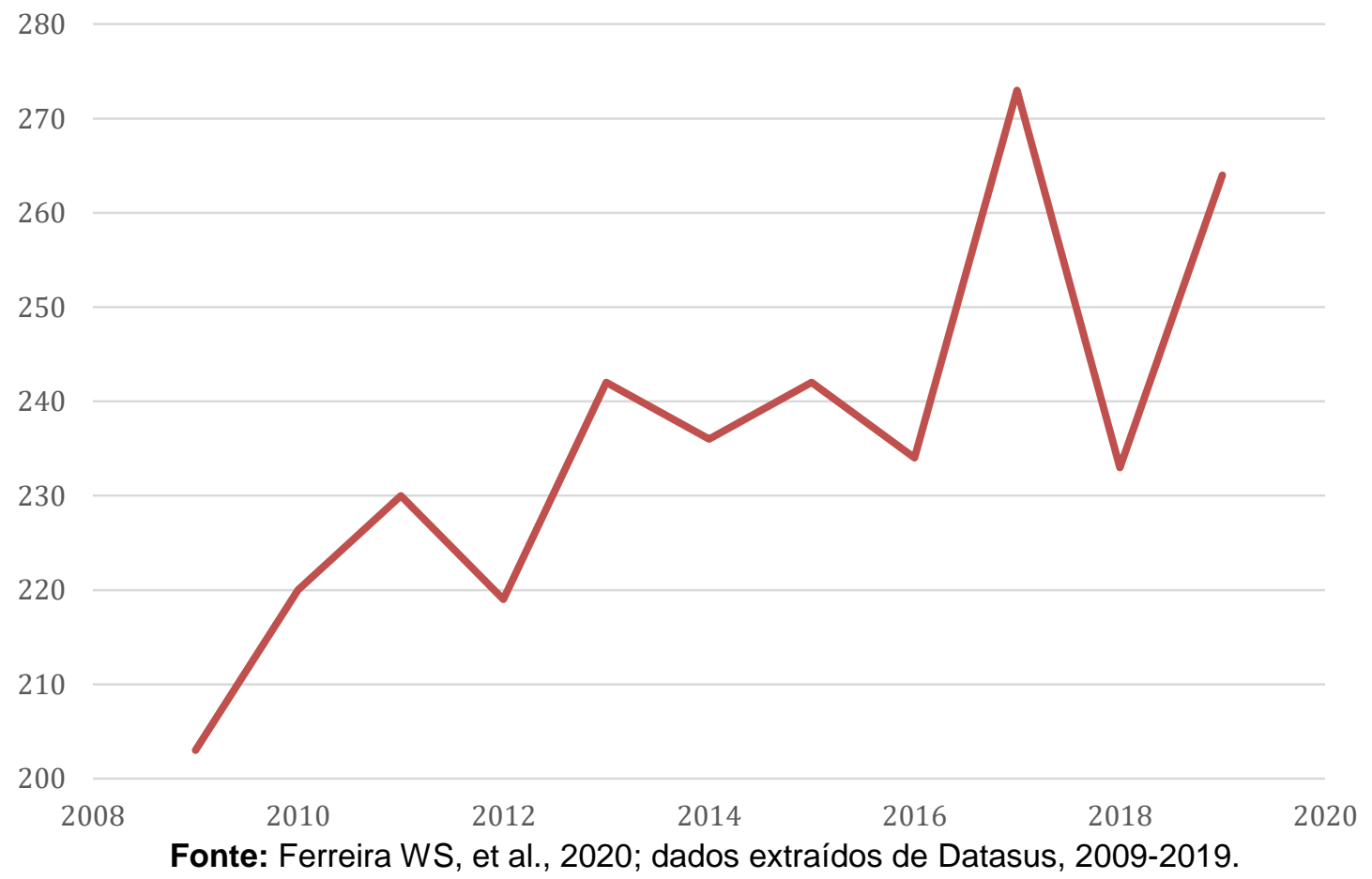


Quanto ao perfil dos coinfectados, o gênero masculino representou a maioria $(69,79 \% ; n=1812)$. As idades variaram entre crianças menores 1 ano a idosos acima de 80 anos. Na faixa etária de 20 a 39 anos, a frequência de coinfecção foi maior $(59,89 \%$; $n=1555)$, seguida pela de 40 a 59 anos $(33,62 \% ; n=873)$. Menores percentuais foram encontrados entre os menores de 1 ano $(0,23 \% ; n=6)$ e naqueles acima de 80 anos $(0,23 \% ; n=6)$ (Tabela 1$)$.

Em relação à raça, $77,65 \%(n=2016)$ das pessoas eram pardas, seguidas de brancos e pretos, com $8,28 \%(n=215)$ e $7,43 \%(n=193)$, respectivamente. A forma clínica pulmonar representou a maioria dos casos, com taxa de notificação de $63,25 \%$ ( $n=1642)$, seguida da TB extrapulmonar com $22,38 \%$ ( $n=581$ ). Em 14,36\% ( $n=373)$ dos casos os indivíduos apresentaram as duas formas da doença (pulmonar e extrapulmonar (Tabela 1).

Tabela 1 - Distribuição do HIV nos casos de Tuberculose em relação ao gênero, faixa etária, raça e forma clínica.

\begin{tabular}{ccc}
\hline Variável & $\mathbf{N}$ & $\%$ \\
\hline Gênero & & \\
\hline Feminino & 784 & 30,20 \\
Masculino & 1812 & 69,79 \\
\hline Faixa etária & & \\
\hline$<1$ Ano & 6 & 0,23 \\
$1-4$ & 7 & 0,26 \\
$5-9$ & 7 & 0,26 \\
$10-14$ & 9 & 0,34 \\
$15-19$ & 42 & 1,61 \\
$20-39$ & 1555 & 59,89 \\
$40-59$ & 873 & 33,62 \\
$60-64$ & 44 & 1,69 \\
$65-69$ & 26 & 1,00 \\
$70-79$ & 21 & 0,80 \\
80 ou mais & 6 & 0,23 \\
\hline Raça & & \\
\hline Parda & 2016 & 77,65 \\
Branca & 215 & 8,28 \\
Amarela & 5 & 0,19 \\
Preta & 193 & 7,43 \\
Indígena & 8 & 0,30 \\
Ignorado/Branco & 159 & 6,12 \\
\hline Forma Clínica & & \\
\hline Pulmonar & 1642 & 63,25 \\
Extrapulmonar & 581 & 22,38 \\
Pulmonar+Extrapulmonar & 373 & 14,36 \\
\hline Total & $\mathbf{2 5 9 6}$ & \\
\hline Fonte:Ferrera WS, & & \\
\hline & 59 & $2009-2019$ \\
\hline
\end{tabular}

Fonte: Ferreira WS, et al., 2020; dados extraídos de Datasus, 2009-2019.

No que se refere à escolaridade, observa-se que a maioria dos coinfectados não completaram o ensino fundamental (32,81\%; $n=852)$, seguido daqueles com ensino médio completo $(16,10 \% ; n=418)$ e com ensino fundamental completo $(10,47 \% ; n=272)$. Os menores índices de coinfecção estão presentes entre os sujeitos com ensino superior incompleto $(1,65 \% ; n=43)$. É importante destacar que 701 casos de notificação $(27,00 \%)$ não apresentavam informações acerca da escolaridade, o que pode subnotificar a categoria (Tabela 2). 
Tabela 2 - Distribuição do HIV nos casos de Tuberculose em relação à escolaridade.

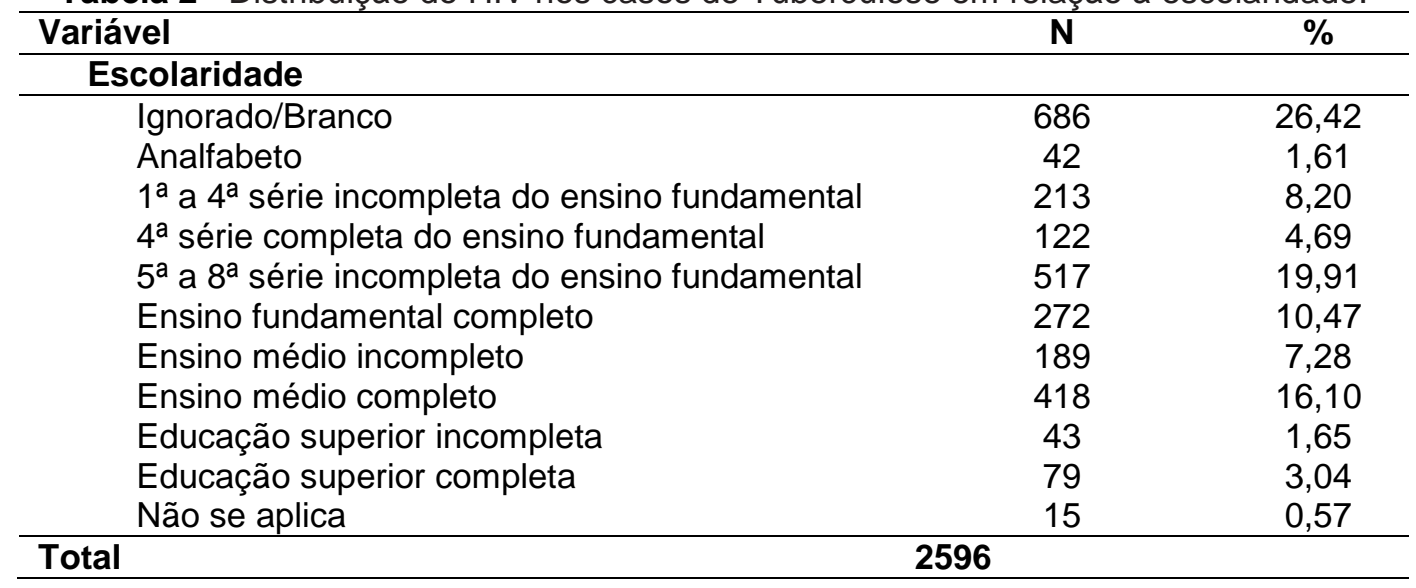

Fonte: Ferreira WS, et al., 2020; dados extraídos de Datasus, 2009-2019.

Dos casos notificados no município de Belém, 79,08\% ( $n=2053)$ foram de casos novos, representando o maior índice em relação ao tipo de entrada. Os casos de reingresso após abandono com 10,01\% $(n=260)$ e de recidiva com $7,28 \%(n=189)$ também são considerados relevantes.É importante ressaltar, ainda, que $51,54 \%$ ( $n=1338$ ) dos sujeitos não estavam sob regime de Tratamento Diretamente Observado (TDO). Ademais, em $34,12 \%(n=886)$ e $71,49 \%(n=1856)$ dos registros, respectivamente, não estavam presentes informações referentes à realização de TDO e da Terapia Antirretroviral (TARV), o que mostra a elevada subnotificação de ambas as categorias. Ao analisar os resultados da situação de encerramento dos casos de TB com coinfecção HIV no município, verificou-se um índice de cura de 46,64\% ( $n=1211)$ e um número significativo de casos de abandono (14,06\%; $n=365)$ e óbito $(20,72 \% ; n=538)$ (Tabela 3).

Tabela 3 - Distribuição do HIV nos casos de Tuberculose em relação ao tipo de entrada, TDO realizado, terapia antirretroviral e situação de encerramento.

\begin{tabular}{lcc}
\hline Variável & $\mathbf{N}$ & $\%$ \\
\hline Tipo de entrada & 2053 & 79,08 \\
\hline Caso novo & 189 & 7,28 \\
Recidiva & 260 & 10,01 \\
Reingresso após abandono & 2 & 0,07 \\
Não sabe & 84 & 3,23 \\
Transferência & 8 & 0,30 \\
Pós óbito & & \\
\hline TDO realizado & 886 & 34,12 \\
\hline Ignorado/branco & 372 & 14,32 \\
Sim & 1338 & 51,54 \\
Não & & \\
Terapia antirretroviral & 1856 & 71,49 \\
Ignorado/branco & 621 & 23,92 \\
Sim & 119 & 4,58 \\
Não & & \\
Situação de encerramento & 296 & 11,40 \\
Ignorado/branco & 1211 & 46,64 \\
Cura & 365 & 14,06 \\
Abandono & 81 & 3,12 \\
Óbito por tuberculose & 457 & 17,60 \\
Óbito por outras causas & 143 & 5,50 \\
Transferência & 23 & 0,88 \\
TB-DR & 19 & 0,73 \\
Mudança de esquema & 1 & \\
Falência & & \\
\hline Total & $\mathbf{2 5 9 6}$ & \\
\hline Fonte: Ferreira WS, et al., & & \\
\hline
\end{tabular}

Fonte: Ferreira WS, et al., 2020; dados extraídos de Datasus, 2009-2019. 
Por fim, a porcentagem de pacientes com TB que não fizeram o teste de infecção pelo HIV diminuiu no decorrer dos anos. Da mesma forma, a porcentagem de pessoas com sorologia conhecida em relação ao número total de casos de tuberculose aumentou de 44,20\% em 2009 para 67,14\% em 2019 (Tabela 4).

Tabela 4 - Realização de teste sorológico para detecção do HIV nos casos de tuberculose.

\begin{tabular}{lcccccc}
\hline $\begin{array}{l}\text { Ano } \\
\text { Diagnóstico }\end{array}$ & Ign/Branco & Positivo & Negativo & $\begin{array}{l}\text { Em } \\
\text { andamento }\end{array}$ & $\begin{array}{l}\text { Não } \\
\text { realizado }\end{array}$ & Total \\
\hline 2009 & - & 203 & 632 & 227 & 827 & 1889 \\
2010 & - & 220 & 799 & 332 & 450 & 1801 \\
2011 & - & 230 & 970 & 285 & 509 & 1994 \\
2012 & - & 219 & 867 & 258 & 481 & 1825 \\
2013 & - & 242 & 930 & 262 & 435 & 1869 \\
2014 & 1 & 236 & 994 & 61 & 485 & 1777 \\
2015 & - & 242 & 846 & 110 & 539 & 1737 \\
2016 & 3 & 234 & 922 & 57 & 516 & 1732 \\
2017 & - & 273 & 846 & 50 & 651 & 1820 \\
2018 & - & 233 & 872 & 29 & 592 & 1726 \\
2019 & - & 264 & 1001 & 150 & 469 & 1884 \\
\hline Total & 4 & 2596 & 9679 & 1821 & 5954 & 20054 \\
\hline
\end{tabular}

Fonte: Ferreira WS, et al., 2020; dados extraídos de Datasus, 2009-2019.

\section{DISCUSSÃO}

Na série histórica de 2009 a 2019, foram registrados 4112 casos da coinfecção TB/HIV no Estado do Pará (SINAN, 2020). Neste intervalo, o município de Belém registrou 2596 casos, abrangendo mais da metade das notificações em todo território paraense, o que pode ser justificado pela alta densidade populacional encontrada na capital.

Ao observar a presença de tuberculose em indivíduos soropositivos em relação ao gênero, percebeu-se que o gênero masculino foi o mais acometido (69,79\%). Tais achados estão em conformidade com estudos realizados em outras regiões do país, como em Salvador, Porto Alegre e Alagoas, onde fora demonstrado um predomínio de $65,0 \%, 64,0 \%$ e $74,2 \%$ dos casos em homens, respectivamente (HAYNE LMP, 2018; SILVA MA, 2018; SANTOS CJ, et al., 2019). Ainda, segundo Castrighini CC, et al. (2017), as razões para maior adoecimento entre os indivíduos do gênero masculino podem ser explicadas por suas características comportamentais, sua maior exposição à doença ou pela maior dificuldade de adesão ao tratamento.

No que tange às características por faixa etária, os indivíduos entre 20 e 39 anos são dominantes $(59,89 \%)$. Esses resultados são consistentes com estudo realizado em um país em desenvolvimento, o qual apontou que a maior proporção de pessoas coinfectadas se encontra na faixa etária economicamente ativa. (RANTI KO, et al., 2016). Nesta perspectiva, ressaltam-se os impactos socioeconômicos de tal associação em termos de afastamento do mercado de trabalho, diminuição da produtividade, perda de renda e gastos relacionados com o tratamento, o que pode aumentar ainda mais a susceptibilidade neste grupo populacional.

Com relação à variável raça, os resultados indicam que os pardos constituem uma proporção expressiva dos coinfectados (77,65\%). Segundo o censo demográfico de 2010 , realizado pelo IBGE, a maior parte da população do município de Belém (64,19\%) autodeclara-se parda. Portanto, pode-se justificar o resultado encontrado no estudo, visto que a amostra populacional é composta predominantemente pela raça parda (IBGE, 2010).

No entanto, resultados diferentes foram encontrados no estudo de Dutra VC (2019), o qual analisou o perfil epidemiológico da coinfecção por tuberculose e HIV nas capitais da Região Sul do país. Neste trabalho, a raça branca teve a maior prevalência de coinfecções. Florianópolis apresentou o maior 
percentual (71,92\%), seguida por Curitiba $(65,36 \%)$ e Porto Alegre $(57,94 \%)$. É imprescindível destacar que de acordo com o censo demográfico realizado pelo IBGE no ano de 2010, a maior parcela da população da região sul $(78,47 \%)$ autodeclara-se branca. Neste sentido, pode-se evidenciar o grande impacto da diversidade étnico-racial brasileira nos resultados de estudos epidemiológicos realizados em diferentes regiões do país.

A forma clínica predominante nos indivíduos coinfectados foi a tuberculose pulmonar $(63,25 \%)$. Conforme o "Manual de Recomendações Para o Controle da Tuberculose no Brasil", a manifestação da tuberculose na forma pulmonar, além de ser a mais recorrente, é também a de maior relevância para a saúde pública, visto que é a forma pela qual a cadeia de transmissão da doença é mantida (BRASIL, 2019).

Quanto ao nível de escolaridade, ressalta-se a frequência da coinfecção em indivíduos com o ensino fundamental incompleto (32,81\%). Resultados semelhantes foram encontrados em outro estudo, onde se verificou que quanto menor o nível de instrução, maior é a frequência da coinfecção por tuberculose e HIV (BRUNELLO ME, et al., 2011). Além disso, segundo Baldan SS, et al. (2017), o nível educacional representa um determinante social da saúde, uma vez que o acesso à educação influencia na capacidade do sujeito em assimilar as informações de promoção de saúde, de prevenção de doenças e na adesão ao tratamento.

Ademais, verificou-se na pesquisa que apenas $14,32 \%$ dos coinfectados estavam sob regime de Tratamento Diretamente Observado (TDO). O tratamento diretamente observado consiste na observação da ingestão dos medicamentos. Essa tarefa é realizada por um profissional treinado, o qual irá acompanhar a tomada da medicação do paciente desde o início do tratamento até a sua cura. Para as pessoas que vivem com HIV, a adesão à terapia medicamentosa é um fator essencial. Desta forma, torna-se de fundamental importância a realização do TDO neste grupo (BRASIL, 2011).

Além do Tratamento Diretamente Observado, é recomendada desde 2011, a Terapia Antirretroviral (TARV) para todas as pessoas com HIV que desenvolvem TB, independentemente da contagem de linfócitos TCD4+. Apesar desta orientação, na maioria dos casos $(71,49 \%)$ os dados referentes à TARV foram ignorados pelos profissionais que notificaram o caso, o que compromete a qualidade da notificação e, consequentemente, da assistência prestada. É importante destacar que a identificação dos indivíduos que aderem ao tratamento antirretroviral é imprescindível, pois a partir do momento em que há falha na adesão, o sistema imunológico estará vulnerável, o que reflete em baixos níveis de linfócitos CD4+, bem como na progressão para a AIDS e o desenvolvimento de doenças oportunistas (SILVA JA, et al., 2015).

Segundo dados nacionais de 2017, pessoas com coinfecção TB/HIV que realizam a TARV e o TDO alcançam uma taxa de cura de $42 \%$ e um percentual de abandono igual a $5 \%$. Para os coinfectados que fazem o uso de TARV, mas não participam do tratamento diretamente observado, a taxa de cura é de $34 \%$ e a de abandono, 13\% (BRASIL, 2018).

No período de realização deste estudo, houve um percentual de casos de TB diagnosticados, nos quais não se realizou o teste anti-HIV $(29,68 \%)$, o que é considerado alto, visto que o "Manual de Recomendações Para o Controle da Tuberculose No Brasil" propõe que todos os pacientes com tuberculose ativa devem ser submetidos ao teste anti-HIV (BRASIL, 2019). Este resultado pode indicar a necessidade de maiores esforços de profissionais da área da saúde e gestores para que todos tenham acesso ao diagnóstico.

Além disso, o Ministério da Saúde preconiza uma taxa de abandono inferior a 5,0\% e uma taxa de cura de $85,0 \%$ para a tuberculose (BRASIL, 2011). Este estudo apontou para uma taxa de cura entre os casos de TB/HIV abaixo do recomendado (46,64\%), assim como taxas de abandono de tratamento elevadas $(14,06 \%)$.

É fundamental destacar, ainda, que em relação ao tipo de entrada no serviço de saúde, os maiores números são de casos novos da coinfecção $(79,08 \%)$. Este dado mostra que apesar de ações instaladas para a contenção da doença, o controle da coinfecção Tuberculose/HIV requer um planejamento que permita reduzir a carga de ambas as enfermidades, com ações articuladas, formando uma rede de atenção integral, eficiente e determinante (BARBOSA IR e COSTAICC, 2014). 
Por fim, apesar das grandes conquistas no tratamento tanto da tuberculose como do HIV, evidencia-se que as duas doenças estão concentradas em áreas de pobreza, onde existem recursos mínimos para o diagnóstico, tratamento e controle da coinfecção. Ainda, ressalta-se que o agravamento das condições sociais e econômicas resulta em uma degradação significativa das condições de vida, aumentando a vulnerabilidade e, consequentemente, o risco de adoecer por tuberculose/HIV (VENDRAMINI SH, et al., 2010).

\section{CONCLUSÃO}

O perfil dos indivíduos acometidos segue o padrão nacional de adoecimento: gênero masculino, raça parda, baixa escolaridade, faixa etária economicamente ativa, forma pulmonar, casos novos e evolução para cura após o tratamento. Todavia, o estudo evidenciou uma baixa adesão ao TDO e à TARV, o que representa um descumprimento às normas recomendadas pelo Ministério da Saúde. Ainda, observou-se um percentual significativo de casos nos quais não se realizou o teste anti-HIV. Sendo assim, evidencia-se a grande importância deste estudo, visto que, conhecer o perfil da população acometida possibilita a implantação de estratégias para a prevenção e controle de ambas as doenças no município.

\section{REFERÊNCIAS}

1. BALDAN SS, et al. Características clínico-epidemiológicas da coinfecção por tuberculose e HIV e sua relação com o Índice de Desenvolvimento Humano no estado do Mato Grosso do Sul, Brasil. Revista Pan-amazônica de Saúde, 2017; 8(3): 59-67.

2. BARBOSA IR, COSTA ICC. Estudo epidemiológico da coinfecção tuberculose-HIV no nordeste do Brasil. Revista de patologia tropical, 2014; 43(1): 1-12.

3. BASTOS SH, et al. Coinfecção tuberculose/HIV: perfil sociodemográfico e saúde de usuários de um centro especializado. Acta Paul Enferm. 2020; 33:1-7.

4. BASTOS SH, et al. Perfil Sociodemográfico e de saúde da coinfecção tuberculose/HIV no Brasil: revisão sistemática. Rev Bras Enferm online, 2019;72(5):1458-1465. Disponível em: https://www.scielo.br/pdf/reben/v72n5/pt_0034-7167reben-72-05-1389.pdf. Acesso em: 13 de jan de 2021.

5. BENITO N, et al. Pulmonary infections in HIV-infected patients: an update in the 21st century. European Respiratory Journal, 2011; 39(3): 730-745.

6. BRASIL. Ministério da Saúde. Secretaria de Vigilância em Saúde. Boletim Epidemiológico: Panorama epidemiológico da coinfecção TB-HIV no Brasil 2019, 50(26), 2019.

7. BRASIL. Ministério da Saúde. Secretaria de Vigilância em Saúde. Departamento de Vigilância das Doenças Transmissíveis. Manual de recomendações para o controle da tuberculose no Brasil/ Ministério da Saúde, Secretaria de Vigilância em Saúde, Departamento de Vigilância das Doenças Transmissíveis. - Brasília: Ministério da Saúde, 2018.

8. BRASIL. Ministério da Saúde. Secretaria de Vigilância em Saúde. Departamento de Vigilância das Doenças Transmissíveis. Manual de recomendações para o controle da tuberculose no Brasil/ Ministério da Saúde, Secretaria de Vigilância em Saúde, Departamento de Vigilância das Doenças Transmissíveis. - Brasília: Ministério da Saúde, 2019.

9. BRASIL. Ministério da Saúde. Secretaria de Vigilância em Saúde. Departamento de Vigilância Epidemiológica. Manual de recomendações para o controle da tuberculose no Brasil / Ministério da Saúde, Secretaria de Vigilância em Saúde, Departamento de Vigilância Epidemiológica. - Brasília: Ministério da Saúde, 2011.

10. BRASIL. Ministério da Saúde. Secretaria de Vigilância em Saúde. Departamento de Vigilância Epidemiológica. Tratamento diretamente observado (TDO) da tuberculose na atenção básica: protocolo de enfermagem / Ministério da Saúde, Secretaria de Vigilância em Saúde, Departamento de Vigilância Epidemiológica. - Brasília: Ministério da Saúde, 2011.

11. BRUNELLO MEF, et al. Áreas de vulnerabilidade para co-infecção HIV-aids/TB em Ribeirão Preto, SP. Revista de Saúde Pública, 2011; 45(3): 556-563.

12. CASTRIGHINI CC, et al. Prevalência e aspectos epidemiológicos da coinfecção HIV/tuberculose. Revista Enfermagem Uerj, 2017; 25: 1-6.

13. DUTRA VC. Perfil epidemiológico da coinfecção tuberculose e HIV nas capitais da região sul no período de 2008 a 2018. TCC (Especialização em Gestão em Saúde) -Escola de Administração. Universidade Federaldo Rio Grande do Sul, Porto Alegre, 2019. 40 p.

14. EFSEN AMW, et al. Major Challenges in Clinical Management of TB/HIV Coinfected Patients in Eastern Europe Compared with Western Europe and Latin America. Plos One, 2015; 10(12): 1-17.

15. HAYNE LMP. Perfil clínico-epidemiológico de coinfecção por tuberculose e HIV no município de Salvador, Bahia, no período de 2008 a 2017. TCC (Graduação em Enfermagem) - Curso de Enfermagem. Universidade Católica do Salvador, Salvador, 2018; 24 p. 
16. IBGE. Instituto Brasileiro de Geografia e Estatística. Censo Demográfico 2010, Área territorial brasileira. 2010. Disponível em: https://www.ibge.gov.br/cidades-e-estados/pa/belem.html. Acesso em: 05 de set de 2020.

17. IBGE. Instituto Brasileiro de Geografia e Estatística. Belém. $2020 . \quad$ Disponível em: https://cidades.ibge.gov.br/brasil/pa/belem/panorama. Acesso em: 05 de set de 2020.

18. MIRANDA LO, et al. Aspectos epidemiológicos da coinfecção Tuberculose/HIV no Brasil: revisão integrativa. Revista Prevenção de Infecção e Saúde, 2017; 3(3):59-70.

19. MONTALES MT, et al. Mycobacterium tuberculosis infection in a HIV-positive patient. Respiratory Medicine Case Reports, 2015; 16:160-162.

20. NEVES LAS, et al.Aids e tuberculose: a coinfecção vista pela perspectiva da qualidade de vida dos indivíduos. Rev Esc Enferm USP, 2012; 46(3): 704-710.

21. OLIVEIRA LB, et al. Análise epidemiológica da coinfecção tuberculose/HIV. Cogitare Enfermagem, 2018; 23(1): 1-8.

22. PAHO. Pan American Health Organization. Tuberculosis in the Americas; Regional Report 2013 (Epidemiology, Control, and Financing). Geneva: WHO; 2014.

23. RANTI KO, et al. Prevalence of HIV infection among tuberculosis patients in a teaching hospital in south-west Nigeria: a four-year retrospective study. HIV \& AIDS Review, 2016; 15(4): 136-140.

24. SANTOS CJ, et al. Aspectos clínicos e epidemiológicos da tuberculose em pacientes com HIV/aids. Medicina (Ribeirão Preto Online), Alagoas, v. 52, n. 3, p. 231-238. Disponível em: http://www.revistas.usp.br/rmrp/article/view/156246. Acesso em: 10 de set de 2020.

25. SILVA JÁ, et al. Fatores associados à não adesão aos antirretrovirais em adultos com AIDS nos seis primeiros meses da terapia em Salvador, Bahia, Brasil. Cadernos de Saúde Pública, 2015; 31(6): 1188-1198.

26. SILVAMA. Coinfecção tuberculose-HIV na cidade de Porto Alegre: análise de dados 2010-2014. Monografia (Especialização em Saúde Pública) - Faculdade de Medicina. Universidade Federal do Rio Grande do Sul, Porto Alegre, 2018; 37p.

27. SOUZA AC, et al. Perfil epidemiológico da coinfecção tuberculose/HIV no período de 2015 a 2019 no Brasil: uma revisão da literatura. Revista Interdisciplinar em Saúde, 2020; 7(único): 563-576.

28. VENDRAMINI SH, et al. Análise espacial da co-infecção tuberculose/HIV: relação com níveis socioeconômicos em município do sudeste do Brasil. Revista da Sociedade Brasileira de Medicina Tropical, 2010; 43(5): 536-541.

29. WHO. World Health Organization. Bendingthe curve - ending TB: Annual report 2017. New Delhi: World Health Organization, 2017.

30. WHO. World Health Organization. Global tuberculosis report 2019. Geneva: World Health Organization, 2019. 